\title{
Enhanced migration of fibroblasts derived from lungs with fibrotic lesions
}

\author{
Hideki Suganuma, Atsuhiko Sato, Ryoji Tamura, Kingo Chida
}

\begin{abstract}
Background - The migration and proliferation of fibroblasts may be important in the pathogenesis of pulmonary fibrosis. Considerable data are available on the proliferation of fibroblasts, but very few on their migration.

Methods - The migratory activity of fibroblasts obtained from lung biopsy specimens from 11 patients with idiopathic pulmonary fibrosis (IPF) was studied using a 96-well chemotaxis chamber. Fibroblasts from eight normal controls, seven patients with interstitial fibrosis associated with a collagen vascular disease (IP-CVD), and 13 patients with sarcoidosis were also examined. Migratory activity was tested in a serum-free medium in the presence and absence of platelet derived growth factor (PDGF), $30 \mathrm{ng} / \mathrm{ml}$, as a chemoattractant.

Results - Migration of fibroblasts from patients with IPF was enhanced in serumfree maintenance medium alone (mean (SD) controls $v$ IPF: 183 (86) $v 689$ (491) cells/field), and was also enhanced when cells were stimulated by PDGF (controls $v$ IPF: 829 (222) $v 1928$ (600) cells/field). Fibroblasts from tissues with dense fibrosis had a greater capacity for migration than those from an earlier stage of fibrosis. No correlation was found between migratory activity and proliferative capacity of the individual cells.
\end{abstract}

Conclusions - The fact that fibroblasts from fibrotic lungs migrate faster than those from controls suggests that migration is related to the initiation of the pulmonary fibrotic process. These in vitro studies suggest that fibroblasts derived from the lungs of patients with pulmonary fibrosis have a migratory phenotype. Such a change in fibroblast phenotype, if it occurred in vivo, may be important in the context of the pathogenesis of pulmonary fibrosis.

(Thorax 1995;50:984-989)

Keywords: migration, fibroblasts, idiopathic pulmonary fibrosis.

Although some types of pulmonary fibrosis are caused by the inhalation of inorganic dusts, allergic reactions, or exposure to radiation, others such as idiopathic pulmonary fibrosis (IPF), IPF associated with collagen vascular disease (IP-CVD), and sarcoidosis have no obvious aetiology.

The mechanism of pulmonary fibrosis has been investigated mainly by analysing the char- acteristics of bronchoalveolar lavage (BAL) cells and the associated histology from patients with pulmonary fibrosis. Histological analysis of pulmonary fibrosis suggests that activation of fibroblasts is an initial step in the process that leads to fibrosis, ${ }^{12}$ and studies of fibroblast function may provide clues to understanding its pathophysiology. Fibroblasts derived from non-fibrosing sites differ in function from those obtained from fibrosing sites with respect to growth activity, ${ }^{3-5}$ production of collagenase or tissue inhibitors of metalloproteinases, ${ }^{6}$ and collagen metabolism. ${ }^{78}$

Intraluminal organisation plays an important part in the progression to pulmonary fibrosis, and one common process is the migration of fibroblasts or myofibroblasts from the interstitial compartment into the air spaces. Fibroblast migration follows injury or inflammation of the epithelial lining cells and their basement membranes. ${ }^{9}$ BAL supernatants from patients with active sarcoidosis or fibrosing alveolitis have an enhanced ability to induce fibroblast migration. ${ }^{1011}$ Others have shown, through analyses of BAL supernatants and cells in fibrotic lungs, that fibronectin, ${ }^{12}{ }^{13}$ collagen, ${ }^{14}$ and platelet-derived growth factor (PGDF) ${ }^{15}$ serve as chemoattractants for fibroblasts. However, no study has focused on migratory activity.

We have investigated the migratory activity of fibroblasts derived from human lungs of patients with IPF, IP-CVD, and sarcoidosis.

\section{Methods}

\section{STUDY POPULATION}

The study population consisted of eight control subjects (four men, mean age 59 years), 11 patients with IPF (eight men, mean age 61 years), seven patients with IP-CVD (two men, mean age 51 years), and 13 patients with sarcoidosis (five men, mean age 44 years). IPF was diagnosed from open lung biopsy specimens, as well as from clinical data and radiological findings. The patients with IP-CVD comprised three cases of scleroderma and four cases of rheumatoid arthritis whose clinical features and laboratory data fulfilled the American Rheumatism Association criteria for each diagnosis. All patients with sarcoidosis were biopsy-proven. Their radiographic grades were as follows: stage 0 , without findings $(n=3)$; stage I, with bilateral hilar lymphadenopathy $(n=3)$; stage II, with bilateral hilar lymphadenopathy and parenchymal infiltrations $(n=4)$; and stage III, solely with parenchymal infiltrations $(n=3)$. Control fibroblasts were established from macroscopically normal areas of lung obtained from eight patients undergoing resection for

The Second Divis Internal Medici

University School of Hamamatsu 431-31, Japan A Sato K Tamura
} 
pulmonary tumours. The tissues from patients with IPF were obtained by open lung biopsy in 10 cases and by transbronchial lung biopsy in one case who had previously been diagnosed as usual interstitial pneumonia by open lung biopsy. To investigate differences between various intensities of fibrosis the open lung biopsy specimens of the patients with IPF were divided into two groups: (1) those with microscopic features predominantly of alveolitis (early fibrosis); and (2) those with more intense lesions of fibrosis (dense fibrosis), according to the classification by Raghu et al. ${ }^{4}$ Five cases of IPCVD had tissue obtained by open lung biopsy which showed histological evidence of interstitial pneumonia. The other two cases of IPCVD and all 13 patients with sarcoidosis had specimens obtained by transbronchial lung biopsy. All patients were informed of the purpose of the study and gave permission for the use of lung specimens.

\section{FIBROBLAST CULTURE}

Lung specimens were minced into pieces smaller than $1 \mathrm{~mm}^{3}$ after removal of extraneous tissue, then washed three times with phosphate buffered saline (PBS) and twice with Dulbecco's modified Eagle's medium (DMEM) (Sigma, St Louis, USA) supplemented with $10 \%$ heat inactivated fetal bovine serum (FBS) (Gibco, Grand Island, New York, USA), 1\% non-essential amino acid solution (Gibco), gentamicin, $30 \mathrm{mg} / \mathrm{l}$ (Wako, Osaka, Japan), and sodium bicarbonate, $0.75 \mathrm{mg} / \mathrm{l}$ (Katayama, Osaka, Japan). The minced and washed tissue fragments were then placed into six-well polystyrene plates (Nunk, Roskilde, Denmark) and covered with a cover slip to make the tissue adhere to the dishes. After the addition of $2 \mathrm{ml}$ of DMEM the tissue was incubated under a humidified atmosphere of $5 \%$ carbon dioxide at $37^{\circ} \mathrm{C}$ with the medium changed twice weekly. When the bottom of each dish was coated with a monolayer of confluent fibroblasts, usually after 4-6 weeks, the cells were detached by the addition of $0.25 \%$ trypsin/ $1 \mathrm{mM}$ EDTA (Gibco), resuspended in DMEM and placed in $75 \mathrm{~cm}^{2}$ flasks (Iwaki, Funahashi, Japan) with $20 \mathrm{ml}$ DMEM. Subsequently, when cells again reached confluency, they were split 1:2. For the following experiments 3-11 $\times$ population doubling level of cells were used for assay. Fibroblast aliquots at different population doubling levels were frozen and stored in liquid nitrogen. The cells had morphological features of fibroblasts by phase contrast microscopic and transmission electron microscopic examination. The cells were confirmed to be free of mycoplasma infection (checked with a MycoTect Kit, Gibco).

\section{MIGRATION ASSAY}

A 96-well chemotaxis chamber apparatus (Neuro Probe, Cabin John, Maryland, USA) with a polyvinyl pyrrolidone-free polycarbonate filter and a pore size of $8 \mu \mathrm{m}$ (Neuro Probe) was used for the migration assay. The volume of each lower chamber was $30 \mu$ and that of each upper chamber was $225 \mu \mathrm{l}$. The exposed filter area was $18 \mathrm{~mm}^{2}$ per well. Since fibroblasts do not normally attach to polycarbonate filters, they were precoated with collagen to allow an adequate number of cells to attach. ${ }^{16}$ Because the collagen with which a filter is coated may possibly influence the migration of fibroblasts, a gelatin-coated filter was used. The results showed that migration was similar to that through a collagen-coated filter (data not shown). It has also been reported that chemotaxis through a chemoattractant-coated filter is not influenced by the chemoattractant. ${ }^{17}$ The filters were immersed in sterile $0.5 \mathrm{M}$ acetic acid at $50^{\circ} \mathrm{C}$ for one hour, rinsed in sterile distilled water, and treated with collagen solution (Bottger, Berlin, Germany), $30 \mu \mathrm{g} / \mathrm{ml}$, overnight at $37^{\circ} \mathrm{C}$, washed with sterile distilled water three times, and air dried. Serum-free DMEM, $30 \mu$, was added to the lower chamber, with or without human recombinant PDGF BB (Amersham) which is known to be a potent inducer of chemotaxis. ${ }^{17}$ The various concentrations of PDGF BB examined were $0 \cdot 3,3,30$, and $100 \mathrm{ng} / \mathrm{ml}$ using a fibroblast line from a control subject. The maximal migratory response was found to be at $30 \mathrm{ng} / \mathrm{ml}$ (data not shown), so this concentration was used in this study. A filter was then placed over each well and the upper chamber was fastened in place.

Detached fibroblasts were harvested by washing the flasks with PBS after their contents had been incubated with $3 \mathrm{ml} 0.25 \%$ trypsin/ 1 mM EDTA for three minutes. The cells were than centrifuged at $800 \mathrm{~g}$ for five minutes and resuspended to $2.5 \times 10^{5}$ cells $/ \mathrm{ml}$ in serum-free DMEM. A $200 \mu$ l aliquot of cell suspension was added to the upper surface of the filter.

To examine the effect of factors that might influence migration, recombinant human interleukin $1 \beta$ (IL-1 $\beta$ ) (Amersham, Buckinghamshire, UK), $1 \mathrm{ng} / \mathrm{ml}$, recombinant human interferon $\gamma$ (IFN- $\gamma$ ) (Genzyme, Cambridge, Massachusetts, USA), $1000 \mathrm{U} / \mathrm{ml}$, indomethacin (Paesel-Rorei, Frankfurt, Germany), $1 \mu \mathrm{g} / \mathrm{ml}$, and dexamethasone (Molecular Probes, Oregon, USA), $1 \mathrm{mmol} / 1$, were each added separately to the cell suspension and the chamber was incubated at $37^{\circ} \mathrm{C}$ for six hours. The filter was collected and its upper surface scraped to remove cells that had not migrated to the lower surface. Fibroblasts that had migrated to the lower surface of the filter were fixed with methanol and stained with May-Gruenwald and Giemsa stains. Migration fibroblasts were counted in two different fields per well at a magnification of $\times 100$. Each assay was performed in triplicate and the mean value was recorded.

\section{PROLIFERATION ASSAY}

Fibroblasts from a confluent monolayer culture were trypsinised and resuspended in DMEM, $5 \times 10^{4}$ cells $/ \mathrm{ml}$. One millilitre of the suspension was delivered to each flat bottomed well of a 12-well polystyrene plate (Corning, New York, USA) and incubated for 24 hours. After the fibroblasts had adhered, the medium was re- 


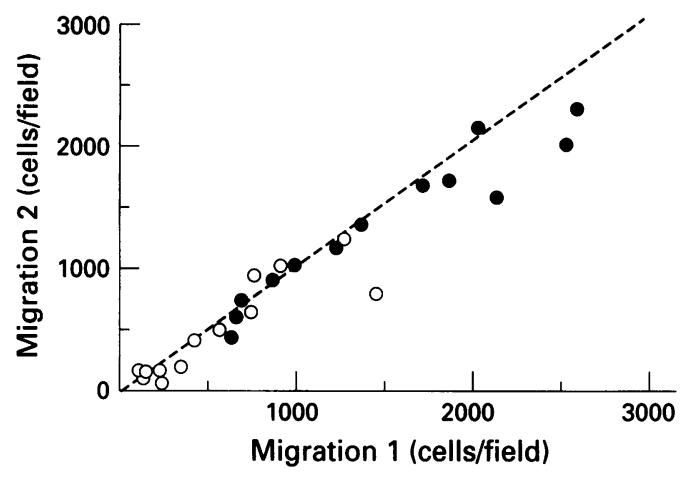

Figure 1 Reproducibility studies. The $x$ axis represents the migration for 13 individual cells from different cases with (O) or without PDGF (O) as a chemoattractant. The $y$ axis represents results for the same cells obtained from repeated experiments. Migration $1=$ results of migration on the first occasion, and migration $2=$ results at the different population doubling levels or after freezing in liquid nitrogen.

moved and replaced with serum-free DMEM. The fibroblasts were incubated for an additional 48 hours to allow the cells to achieve proliferative quiescence. The medium was then exchanged with $1 \mathrm{ml}$ of fresh DMEM and the number of fibroblasts was counted with a haemocytometer (day 0 ). The mean (SD) count at day 0 was $9 \cdot 1(3 \cdot 1) \times 10^{4}$ in controls, $9.3(4.3) \times 10^{4}$ in patients with IPF, 6.7 $(2 \cdot 1) \times 10^{4}$ in patients with IP-CVD, and $6 \cdot 3$ $(2 \cdot 4) \times 10^{4}$ in patients with sarcoidosis. After subsequent incubation for 24,48 , and 96 hours the number of cells was again counted (days 1,2 , and 4 , respectively). These values were
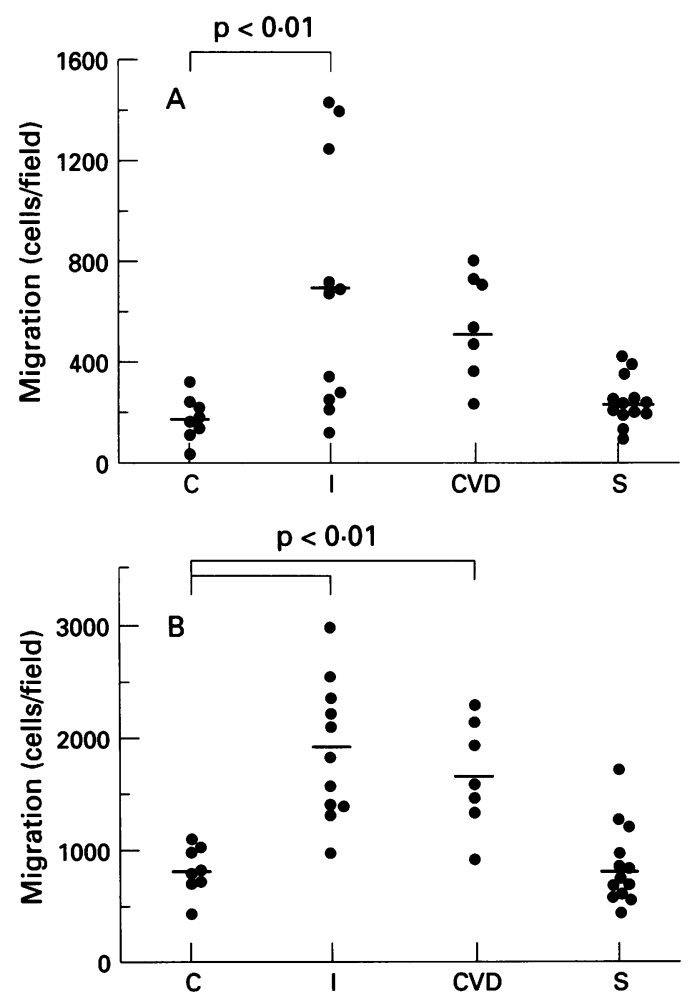

Figure 2 Migration of fibroblasts derived from lung tissue from controls (C), and from patients with IPF $(I), I P$ from controls $(C)$, and from patients with IPF $(I), I P-$
$C V D(C V D)$, and sarcoidosis $(S)$ both without $(A)$ and with (B) PDGF as a chemoattractant. Each bar represents the mean value for each group. expressed as a proliferation ratio to the number of cells at day 0 . Each test was performed in triplicate and the mean value was recorded.

SYNTHESIS OF INTERLEUKIN $1 \alpha$ AND HYALURONAN BY FIBROBLASTS

Fibroblasts at confluency were incubated in serum-free DMEM in 12-well polystyrene plates (Corning, New York, USA) for 48 hours. One millilitre of medium from each well was then replaced with DMEM containing $10 \%$ FBS. After incubation for 24 hours the supernatants were extracted. Concentrations of IL- $1 \alpha$ in the fibroblast supernatants were assayed with an enzyme linked immunosorbent assay kit (Amershan, Buckinghamshire, UK) and hyaluronan concentrations were assayed with a hyaluronic acid binding protein assay kit (Chyugai, Tokyo, Japan).

STATISTICAL ANALYSIS

Data were analysed with a Macintosh computer, using the Statview 4.0 Statistical Package (Abcus Concepts Inc, Berkeley, California, USA). Analysis of variance was performed with Scheffe's test, and the Student's $t$ test for paired samples was applied to the data when appropriate. A $p$ value of $<0.05$ for a difference between groups was considered significant.

\section{Results}

REPRODUCIBILITY OF MIGRATION ASSAY

An interassay was performed to assess the reproducibility of migration by examining 13 fibroblasts from three controls, five patients with IPF, and five patients with sarcoidosis. Fibroblasts with a wide range of migration were chosen and the migratory activities at different population doubling levels were studied. Fibroblasts that had been kept frozen in liquid nitrogen for several weeks were also used. The results are shown in fig 1 . Migration 1 was the result of the first migration study, and migration 2 was the result at the different population doubling levels or after freezing in liquid nitrogen. The coefficient of correlation between the two different conditions was 0.96 , showing no effect on migration of population doubling level or storage of fibroblasts.

MIGRATORY CHARACTERISTICS OF FIBROBLASTS As shown in fig 2, fibroblasts from lung tissue of patients with IPF or IP-CVD had a higher level of migration capacity than those from normal subjects, both in the presence and absence of PDGF as a chemoattractant. The mean (SD) migration of fibroblasts in serumfree maintenance medium (DMEM) alone was 183 (86) cells/field in controls, 689 (491) in patients with IPF, 517 (238) in patients with IPCVD, and 227 (99) in patients with sarcoidosis. The migration of cells with PDGF was 829 (222), 1928 (600), 1687 (478), and 862 (358) cells/field in controls and patients with IPF, IPCVD, and sarcoidosis, respectively. 


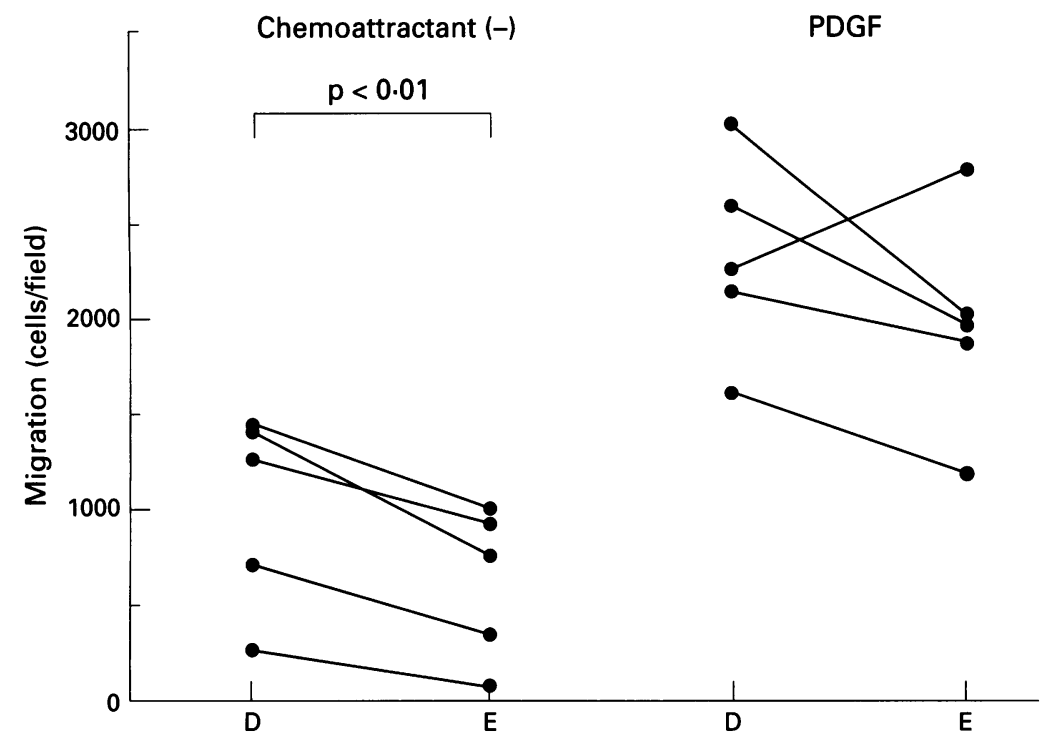

Figure 3 Differences between fibroblasts derived from areas of dense fibrosis (D) and early fibrosis (E), with or without PDGF (30 ng/ml) as a chemoattractant, for five patients with IPF. Mean values are plotted.

To determine the effect of the origin of fibroblasts with various degrees of fibrotic changes on migrations, the migration of fibroblasts derived from early fibrosis and from dense fibrosis was compared (fig 3). The migration of fibroblasts from areas of dense fibrosis was greater than that of fibroblasts from early fibrosis, with the exception of one case stimulated with PDGF. No differences in migration were found for fibroblasts from patients with sarcoidosis of different radiographic grades (data not shown).

The effects of IL- $1 \beta$, IFN- $\gamma$, indomethacin, and dexamethasone on the migration of fibroblasts from each type of pulmonary fibrosis were investigated (table 1). No evidence of chemokinetic activity was seen, but fibroblast migration was somewhat inhibited by IL-1 $\beta$ and dexamethasone. There were no statistically significant differences in fibroblast migration among controls and patients with IPF, IPCVD, and sarcoidosis.

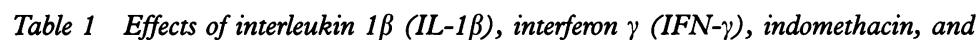
dexamethasone on migration of fibroblasts

\begin{tabular}{|c|c|c|c|c|c|c|}
\hline & No. & $\begin{array}{l}\text { Control values } \\
\text { (mean (SD) } \\
\text { cell number) }\end{array}$ & $\begin{array}{l}I L-1 \beta \\
(\%)\end{array}$ & $\begin{array}{l}I F N-\gamma \\
(\%)\end{array}$ & $\begin{array}{l}\text { Indomethacin } \\
(\%)\end{array}$ & $\begin{array}{l}\text { Dexamethasone } \\
\text { (\%) }\end{array}$ \\
\hline $\begin{array}{l}\text { Controls } \\
\text { IPF } \\
\text { IP-CVD } \\
\text { Sarcoidosis }\end{array}$ & $\begin{array}{l}5 \\
8 \\
5 \\
5\end{array}$ & $\begin{array}{l}251(114) \\
782(540) \\
524(195) \\
282(193)\end{array}$ & $\begin{array}{l}66 \cdot 6(39 \cdot 6) \\
57 \cdot 3(12 \cdot 3) \\
64 \cdot 1(19 \cdot 2) \\
70 \cdot 6(15 \cdot 8)\end{array}$ & $\begin{array}{r}105.2(38.9) \\
96.2(26.2) \\
91.2(21.4) \\
93.0(20.7)\end{array}$ & $\begin{array}{l}98.8(37.1) \\
83.4(28.6) \\
81.5(23.4) \\
74.8(15.8)\end{array}$ & $\begin{array}{l}66.7(20.3) \\
75.8(12.7) \\
71 \cdot 2(19.4) \\
74.1(13.4)\end{array}$ \\
\hline
\end{tabular}

Control values are mean (SD) migrated cells with only the serum-free medium. Other results are expressed as mean (SD) percentage of control values.

IL $-1 \beta, 1 \mathrm{ng} / \mathrm{ml}$, IFN- $\gamma, 1000 \mathrm{U} / \mathrm{ml}$, indomethacin, $1 \mu \mathrm{g} / \mathrm{ml}$, and dexamethasone, $1 \mathrm{mmol} / \mathrm{l}$, were each added separately to the cell suspension.

Table 2 Mean (SD) in vitro fibroblast proliferation ratio in various interstitial lung diseases compared with day 0

\begin{tabular}{lclll}
\hline & No. & Day 1 & Day 2 & Day 4 \\
\hline Controls & 6 & $1 \cdot 10(0 \cdot 28)$ & $2 \cdot 24(1 \cdot 09)$ & $5 \cdot 62(1 \cdot 35)$ \\
IPF & 4 & $1 \cdot 36(0 \cdot 36)$ & $3 \cdot 12(0.98)$ & $7 \cdot 56(3.65)$ \\
IP-CVD & 4 & $1 \cdot 45(0 \cdot 26)$ & $2.98(0 \cdot 68)$ & $9 \cdot 00(4 \cdot 77)$ \\
Sarcoidosis & 10 & $1 \cdot 80(0 \cdot 26)^{*}$ & $3.66(0.72)^{* *}$ & $8 \cdot 70(2 \cdot 44)$ \\
\hline
\end{tabular}

${ }^{*} \mathrm{p}<0.01,{ }^{* *} \mathrm{p}<0.05$ versus controls (ANOVA and Scheffe's test).
PROLIFERATION OF FIBROBLASTS

Compared with fibroblasts from controls, fibroblasts derived from patients with fibrosing pulmonary tissues, especially in sarcoidosis, showed a tendency towards increased proliferation (table 2). No correlation was found between migration (cells/field) and proliferation of fibroblasts derived from any patient (six controls, four IPF, four IP-CVD, and 10 sarcoidosis), whether stimulated with PDGF or not.

To evaluate the characteristics of protein synthesis by fibroblasts from fibrotic lungs, the concentrations of IL- $1 \alpha$ and hyaluron were assayed in the maintenance medium in which fibroblasts were cultured in a confluent monolayer. Interleukin- $1 \alpha$ could not be detected with an ELISA kit (the minimum detectable concentration was $3.9 \mathrm{pg} / \mathrm{ml}$ ). The mean (SD) hyaluronan levels were raised at $3980(680) \mathrm{ng} /$ $\mathrm{ml}$ in controls, $4230(1680) \mathrm{ng} / \mathrm{ml}$ in patients with IPF, $3920(1800) \mathrm{ng} / \mathrm{ml}$ in patients with IP-CVD, and $4560(370) \mathrm{ng} / \mathrm{ml}$ in patients with sarcoidosis, compared with maintenance medium with $10 \%$ FBS $(40 \mathrm{ng} / \mathrm{ml})$. However, the hyaluronan levels were all similar to each other.

MORPHOLOGICAL FEATURES OF FIBROBLASTS Fibroblasts, whether derived from non-fibrotic or fibrotic lungs, were typically spindle-shaped upon phase contrast microscopic examination. Because the number of intracellular filaments in fibroblasts is considered to be closely related to cell mobility, ${ }^{18}$ the number of actin filaments in cultured fibroblasts from patients with IPF were compared with that in control fibroblasts by electron microscopy, but no differences were found.

\section{Discussion}

The fibrosing process centres on various functions of fibroblasts which can act as either "effector cells" or "target cells". ${ }^{19}$ Studying the characteristics of fibroblasts helps to explore the mechanisms by which lungs become fibrotic.

The present study sought to ascertain whether fibroblasts from fibrotic lungs have an enhanced migratory activity related to the development of fibrosis. We found that fibroblasts from patients with IPF showed an increased capacity for migration which was proportional to the intensity of the fibrosis. More interestingly, migration had no relation to the proliferative activity of fibroblasts. Fibroblasts from patients with IP-CVD also showed a tendency towards increased migration, whereas fibroblasts from patients with sarcoidosis showed the same level of migration as control fibroblasts. This result supports the fact that less fibrosis develops in sarcoidosis than in IPF or IP-CVD, but it may also reflect different pathogeneses for these diseases.

Fibroblast function was studied using fibroblasts cultured from both open lung biopsy and transbronchial lung biopsy specimens. Fibroblasts from transbronchial lung biopsy specimens had a higher population doubling level 
than those from open lung biopsy specimens as the transbronchial lung biopsy specimens were smaller. Fibroblast migration was therefore studied at different population doubling levels but no differences were found.

Although fibroblast migration may result from activation, several other factors and diseases are known to affect the response of fibroblasts to chemoattractants. Tumour derived or virus transformed cells possess a greater migratory potential than normal fibroblasts. ${ }^{20}$ Fibroblasts derived from patients with mucopolysaccharidosis or with premature ageing syndrome (progeria) tend to have a reduced chemotactic response when compared with fibroblasts from normal controls. ${ }^{21}$ With respect to receptors on fibroblasts for a chemoattractant, enhanced migration of fibroblasts from our patients with IPF was noted with or without PDGF as a chemoattractant, suggesting that enhanced migration is not totally mediated by alterations in PDGF receptors.

Because of the enhanced migration of fibroblasts from patients with IPF, we initially speculated that protein synthesis might be altered in these cells in culture or even in vivo. It has been reported that IL- $1 \alpha$ present on fibroblasts induces an enhancement of chemotactic responsiveness without any change in PDGF binding to fibroblasts, ${ }^{23}$ and that fibroblasts can produce IL- $1 \alpha^{24}$ Some proteins such as elastin, ${ }^{25}$ hyaluronan, ${ }^{26}$ collagen, ${ }^{27}$ and gelsolin $^{28}$ are also thought to be related to fibroblast migration. We examined the concentrations of $\mathrm{IL}-1 \alpha$ and hyaluronan in fibroblast cultures but were not able to show any difference in concentration of either substance in fibroblasts of different origins.

Alteration of the intracellular filaments composed of actin and myosin may be related to the enhanced migration of fibroblasts from patients with IPF. Cell movement is thought to be affected by the intracellular content of actin filaments and by polymerisation of actin. ${ }^{18}$ The actin content increases in fibrotic lungs, ${ }^{29-31}$ but electron microscopic examination failed to reveal any obvious differences between intracellular filaments from control fibroblasts and those from patients with IPF.

We also examined the response to agents that mediate intracellular signal transduction in conjunction with the migration of fibroblasts. Cellular mechanisms of chemotactic migration include biological changes in responsive cells. ${ }^{32}$ Factors that affect intracellular signal transduction pathways, such as IL-1 $\beta$ and dexamethasone, suppressed the migration of fibroblasts, but the degree of suppression did not differ for fibroblasts from patients with various lung diseases. We deduce that high concentrations of IL-1 $\beta$ maintain the accumulation of fibroblasts and that steroids inhibit fibroblast migration. Further examination will be necessary, however, including examination of a dose-response relationship for these factors. We were unable to explain the enhanced capacity for migration of fibroblasts from patients with IPF.

Fibroblast migration may result from injury or inflammation of the epithelial lining cells and their basement membranes. Undetermined agents may be associated with the enhanced migration of fibroblasts from patients with IPF. Although the mechanism of such migration is not fully understood, studies on the role of such enhanced migration may help in the understanding of the pathogenesis of interstitial lung disease.

1 Adler KB, Callahan LM, Evans JN. Cellular alterations in the alveolar wall in bleomycin-induced pulmonary fibrosis in rats. Am Rev Respir Dis 1986;133:1043-8.

2 Katzenstein AA. Pathogenesis of fibrosis in interstitial pneumonia. Hum Pathol 1985;16:1015-24.

3 Jordana M, Schulman J, McSharry C, Irving LB, Newhouse MT, Jordana G, et al. Heterogeneous proliferative characteristics of human adult lung fibroblast lines and clonally-derived fibroblasts from control and fibrotic human tissue. Am Rev Respir Dis 1988;138:579-84.

4 Rague G, Chen Y, Rusch V, Rabinovitch PS. Differential proliferation of fibroblasts cultured from normal and fibproticeration of fibroblasts cultured from normal

5 Mio T, Nagai S, Kitaichi M, Kawatani A, Izumi T. Proliferative characteristics of fibroblast lines derived from open lung biopsy specimens of patients with IPF(UIP). Chest 1992;102:832-7.

6 Pardo A, Selman M, Ramirez R, Ramos C, Montano M, Stricklin G, et al. Production of collagenase and tissue inhibitor of metalloproteinases of fibroblasts derived from normal and fibrotic human lungs. Chest 1992;102:1085-9.

7 Narayanan AS, Whithey J, Souza A, Rague G. Effect of $\gamma$ interferon on collagen synthesis by normal and fibrotic interferon on collagen synthesis by normal and fibr

8 Harrison NK, Argent AC, McAnulty RJ, Black CM, Corrin $B$, Laurent GJ. Collagen synthesis and degradation by systemic sclerosis lung fibroblasts. Chest $1991 ; 99: 71 \mathrm{~s}-2 \mathrm{~s}$.

9 Basset F, Ferrans VJ, Soler P, Takemura T, Fukuda Y, Crystal RG. Intraluminal fibrosis in interstitial lung disorders. Am $\mathcal{F}$ Pathol 1986;122:443-61.

10. Weber J, Meyer KC, Banda P, Calhoun WJ, Auerbach R. Studies of bronchoalveolar lavage cells and fluids inn pulmonary sarcoidosis. Am Rev Respir Dis 1989;140:14504 .

11 Behr J, Adelmann-Grill BC, Krombach F, Beinert $T$, Schwaiblmair M, Fruhmann. Fibroblast chemotactic reschwaiblmair $M$, Fruhmann. Fibroblast chemotactic response elicited by native bronchoalveolar lavage fluid from
patients with fibrosing alveolitis. Thorax 1993;48:736-42.

12 Rennard SI, Crystal RG. Fibronectin in human bronchopulmonary lavage fluid. $\mathcal{f}$ Clin Invest 1981;69:113-22.

13 Rossi GA, Bitterman PB, Rennard SI, Ferrans VJ, Crystal RG. Evidence for chronic inflammation as component of the interstitial lung disease associated with progressive systemic sclerosis. Am Rev Respir Dis 1985;131:612-7.

14 Low RB, Giancola MS, King TE, Chapitis J, Vacek P, Davis GS. Serum and bronchoalveolar lavage of $\mathrm{N}$-terminal type III procollagen peptides in idiopathic pulmonary fibrosis. III procollagen peptides in idiopathic

15 Shaw RJ, Benedict SH, Clark RAF, King TE. Pathogenesis of pulmonary fibrosis in interstitial lung disease. Am Rev Respir Dis 1991; 143:167-73.

16 Postlethwaite AE, Snyderman R, Kang AH. The chemotactic attraction of human fibroblasts to a lymphocytederived factor. $\mathcal{F} \operatorname{Exp}$ Med 1976;144:1188-203.

17 Adelmann-gill BC, Cully Z. Signal perception of fibroblasts for directional migration to platelet-derived growth factor
in Boyden-type chambers. $\mathscr{f}$ Cell Physiol 1990;143:172-7.

18 Hartwig JH. Mechanisms of cell movement. In: Crystal RG, West JB, eds. The lung; scientific foundations. New York: West JB, eds. The lung; scien
Raven Press, 1991: 141-53.

19 Rochester CL, Elias JA. Cytokines and cytokine networking in the pathogenesis of interstitial and fibrotic lung disorders. Sem Respir Med 1993;14:389-416.

20 Mensing H, Albini A, Krieg T, Pontz BF, Müller PK Enhanced chemotaxis of tumour-derived and virus-transformed cells to fibronectin and fibroblast-conditioned medium. Int $\mathcal{f}$ Cancer 1984;33:43-8.

21 Pontz BF, Albini A, Mensing H, Cantz M, Müller PK. Pattern of collagen synthesis and chemotactic response of fibroblasts derived from mucopolysaccharidosis patients. Exp Cell Res 1984;155:457-66.

22 Albini A, Pontz BF, Mensing H, Pilz C, Müller PK. Fibroblast-conditioned medium as chemoattractant: deteriorating response of senescent human fibroblasts and progeroid cells. Hoppe Seyler's Z Physiol Chem 1984;365: proge.

23 Heckmann M, Adelmann-Grill BC, Hein R, Krieg T. Biphasic effects of interleukin-1 $\alpha$ on dermal fibroblasts: enhancement of chemotactic responsiveness at low concentrations and of mRNA expression for collagenase a high concentrations. F Invest Dermatol 1993;100:780-4.

24 Elias JA. Reynolds MM. Interleukin-1 and tumor necrosis factor synergistically stimulate lung fibroblast interleukin 1a production. Am ₹ Respir Cell Mol Biol 1990;3:13-20.

25 Mecham RP, Griffin GL, Madaras JG, Senior RM. Appearance of chemotactic responsiveness to elastin peptides by developing fetal bovine ligament fibroblasts parallels the onset of elastin production. $F \mathrm{Cell} \mathrm{Biol} \mathrm{1984;98:1813-6.}$ 
26 Elias I, Grey M, Schor AM, Schor SL. Antagonistic effects of TGF- $\beta$ and MSF on fibroblast migration and hyaluronic acid synthesis. F Cell Sci 1992;102:447-56.

27 Mallein-Gerin F, Garrone R, Rest M. Proteoglycan and collagen synthesis are correlated with actin organization in differentiating chondrocytes. Eur $\mathcal{F}$ Cell Biol 1991;56: in different

28 Cunningham CC, Stossel TP, Kwiatkowski DJ. Enhanced motility in NIH 3T3 fibroblasts that overexpress gelsolin. Science 1991;251:1233-6.

29 Adler KB, Craighead JE, Vallyathan NV, Evans JN. Actin- containing cells in human pulmonary fibrosis. Am $\mathcal{F}$ Pathol 1981;102:427-37.

30 Low RB, Woodcock-Mitchel J, Evans JN, Adler KB. Actin content of normal and bleomycin-fibrotic rat lung. $\mathrm{Am}$ Rev Respir Dis 1984;129:311-6.

31 Kuhn C, McDonald JA. The role of the myofibroblast in idiopathic pulmonary fibrosis. Am $\mathcal{f}$ Pathol 1991;138: idiopathic $1257-65$.

32 Senior RM, Daughaday GC. Chemotaxis. In: Crystal RG, West JB, eds. The lung; scientific foundations. New York: Raven Press, 1991: 131-9. 